PSICOLOGÍA

IBEROAMERICANA
Psicología Iberoamericana ISSN: 1405-0943

revista.psicologia@ibero.mx

Universidad Iberoamericana, Ciudad de México México

\title{
Evaluación y atención en línea de problemas de salud mental durante la pandemia de Covid-19
}

Pedroza-Cabrera, Francisco Javier; Martínez-Martínez, Kalina Isela; Carranza-Jasso, Rodrigo; Salvador López-Salas, Juan

Evaluación y atención en línea de problemas de salud mental durante la pandemia de Covid-19

Psicología Iberoamericana, vol. 29, núm. 3, Esp., e293324, 2021

Universidad Iberoamericana, Ciudad de México, México

Disponible en: https://www.redalyc.org/articulo.oa?id=133968747006

DOI: https://doi.org/10.48102/pi.v29i3.324

\section{(c) (1)}

Esta obra está bajo una Licencia Creative Commons Atribución 4.0 Internacional. 
Psicología Iberoamericana, vol. 29, núm. 3, Esp., e293324, 2021

Universidad Iberoamericana, Ciudad de México, México

Recepción: 19 Enero 2021

Aprobación: 22 Junio 2021

DOI: https://doi.org/10.48102/ pi.v29i3.324

Redalyc: https://www.redalyc.org/ articulo.oa?id=133968747006

Financiamiento

Fuente: Universidad Autónoma de

Aguascalientes

$\mathrm{N}^{\circ}$ de contrato: PIPS20-8C

Beneficiario: Francisco Javier Pedroza-

Cabrera
Artículos

\section{Evaluación y atención en línea de problemas de salud mental durante la pandemia de Covid-19}

Online assessment and care of mental health problems during the COVID-19 pandemic

\author{
Francisco Javier Pedroza-Cabrera fjpedroz@correo.uaa.mx \\ Universidad Autónoma de Aguascalientes, México \\ iD https://orcid.org/0000-0003-4280-3795 \\ Kalina Isela Martínez-Martínez kimartin@correo.uaa.mx \\ Universidad Autónoma de Aguascalientes, México \\ (D) https://orcid.org/0000-0002-3250-1809 \\ Rodrigo Carranza-Jasso rodrigo.carranza@edu.uaa.mx \\ Universidad Autónoma de Aguascalientes, México \\ (D) https://orcid.org/0000-0001-5105-2466 \\ Juan Salvador López-Salas salvador.lopez@edu.uaa.mx \\ Universidad Autónoma de Aguascalientes, México \\ iD https://orcid.org/0000-0001-9981-5733
}

Resumen: En el presente estudio cuantitativo se evaluaron remotamente problemas de salud mental relacionados con ansiedad, depresión, riesgo suicida, consumo de sustancias y violencia. Se identificó el efecto del confinamiento por pandemia en la población de Aguascalientes, por medio de un diseño descriptivo transversal. Se contó con 4,344 participantes de entre 12 y 60 años de la Línea VIVE referidos por un chatbot de evaluación psicológica, que también retroalimenta el nivel de riesgo y ofrece atención psicológica remota. Se evalúo remotamente el riesgo suicida, depresión y ansiedad y consumo de sustancias en tres fases, una previa y dos durante la cuarentena. Estas tasas presentaron una tendencia a incrementar conforme la pandemia evolucionó. Se encontraron diferencias estadísticamente significativas aplicando el estadístico Kruskal Wallis. Los resultados mostraron que la cuarentena impactó en la salud mental de las personas, al grado de requerir algún tratamiento psicológico. Finalmente, resulta recomendable fortalecer los servicios de internet y el uso de dispositivos electrónicos inteligentes para proveer servicios de salud mental en línea e incrementar la búsqueda de atención por parte de la población.

Palabras clave: ansiedad, consumo de sustancias, COVID-19, depresión, violencia.

Abstract: Mental health problems related to anxiety, depression, suicide risk, substance use and violence were remotely evaluated in the present quantitative study. The effect of confinement due to the pandemic in the Aguascalientes population in Mexico was identified through a descriptive cross-sectional design. There were 4,344 participants between the ages of 12 and 60 years old from the VIVE Line referred by a psychological evaluation chatbot, which also provides feedback on the level of risk and offers remote psychological care. Suicidal risk, depression and anxiety, and substance use were remotely assessed in three phases, one prior and two during quarantine. These rates tended to increase as the pandemic evolved. Statistically significant differences were found applying the Kruskal Wallis statistic. The results showed that the quarantine had an impact on people's mental health, to the extent of requiring some psychological 
treatment. Finally, it is advisable to strengthen internet services and the use of smart electronic devices to provide mental health services online and increase the population's search for care.

Keywords: anxiety, COVID-19, depression, substance consumption, violence.

\section{Introducción}

COVID-19 o enfermedad por coronavirus 2019, es causada por el SARS-CoV-2, altamente infecciosa y posee un período prolongado de incubación ( $\mathrm{Li}$ et al., 2020). Este invasivo virus tuvo su origen en Hubei, provincia de Wuhan a finales del 2019, creciendo exponencialmente el número de casos por todo China hasta extenderse por el mundo entero (Ho et al., 2020). El brote del COVID-19 se convirtió en una emergencia de salud pública de índole internacional a partir del 30 de enero de 2020, de acuerdo con la Organización Mundial de la Salud (OMS), cuando las 34 ciudades de China tuvieron casos de infección, incluso, sin haber tenido contacto directo con los casos iniciales. Pero fue hasta el de 11 de marzo de 2020 que la OMS lo reconoció como una pandemia.

En México el primer caso de contagio positivo del COVID-19 se presentó el 27 de febrero de 2020, y la primera muerte asociada a la enfermedad se presentó el 18 de marzo (BBC, 2020). Sin embargo, tres días antes, el 17 de marzo de 2020, la Secretaría de Salud Federal anunció la implementación de la Jornada Nacional de Sana Distancia como medida preventiva (Gobierno de México, 2020). De esta manera, se suspendieron temporalmente las actividades no esenciales y se reprogramaron los eventos de concentración masiva. Igualmente, se adelantó el periodo vacacional de Semana Santa en las escuelas. El 24 de marzo se declaró la fase dos de la pandemia, y el 30 de marzo se declaró un estado de Emergencia Sanitaria en todo el país, por lo que la Jornada Nacional de Sana Distancia se extendió hasta el 30 de abril. El estado de emergencia sanitaria implicó suspender totalmente las actividades no esenciales. No obstante, en México no se estableció un encierro obligatorio ni un toque de queda, únicamente se persuadió a la población para que permaneciera en su casa el mayor tiempo posible y acatara las medidas de higiene implementadas por la Secretaría de Salud. El 16 de abril el Gobierno Federal extendió la Jornada de Sana Distancia un mes más, es decir hasta el 30 de mayo, sin embargo, el 22 de abril México entró en la fase tres de la pandemia. A partir del 30 de mayo se declaró la "nueva normalidad" y el regreso a las actividades se dió con base en una semaforización epidemiológica de acuerdo con la dispersión del virus.

En acuerdo con los datos de la Universidad Johns Hopkins (2020) sobre el COVID-19, el 29 de junio se reportaron 10,143,794 personas contagiadas y 502,551 muertes asociadas al coronavirus a nivel mundial. Siendo Estados Unidos de América el país que reporta el mayor número de contagios con 2,543,270 personas y 125,660 muertes. El Instituto Mexicano del Seguro Social (IMSS, 2020) al mismo día reportó 220,657 
casos confirmados y 27,121 muertes, en dicho reporte, respecto al Estado de Aguascalientes, se reportaron 2990 casos positivos y 151 defunciones.

Actualmente, en todo el mundo se vive una experiencia sin precedente, que moviliza todas las instituciones de gobierno, principalmente las sanitarias, y organizaciones de la sociedad civil para enfrentar la pandemia del COVID-19. Para contener el contagio entre la población, la OMS sugirió el aislamiento social, limitación de la movilidad o cuarentena de la población, lo que da como resultado problemas emocionales que incrementan la posibilidad de presentar, por primera vez, problemas mentales, o la exacerbación de los trastornos mentales preexistentes (Caballero-Domínguez \& Campo-Arias, 2020). De la misma manera, la pandemia por el COVID-19 tiene un impacto psicológico en la población general con consecuencias en la salud física y mental, caracterizado por la incertidumbre, ira, aumento del consumo de alcohol o de cigarrillos, aislamiento social y el desarrollo del trastorno de estrés post traumático (Shigemura et al., 2020). Un ejemplo de lo anterior es una encuesta que se realizó en China, a 1,210 personas mostró que el $53.8 \%$ valoro\# que la pandemia tuvo un impacto psicológico de moderado a grave, $16.5 \%$ refirió síntomas depresivos entre moderados a graves, $28.8 \%$ síntomas de ansiedad entre moderados a graves y un $8.1 \%$ niveles de estrés entre moderados a graves (Ho et al., 2020). Así mismo, en China se realizó un estudio para evaluar los efectos del confinamiento en menores de edad, encontrando que más de un $80 \%$ de los encuestados paso\# entre 20 y 24 horas al día confinados en casa y la principal preocupación (75.2\%) fue que sus familiares se contagiaran de COVID-19 (Wang et al., 2020).

En un estudio que considera las repercusiones psicológicas derivadas de las pandemias, se realiza un metaanálisis tanto de la influenza $\mathrm{H} 1 \mathrm{~N} 1$ como el COVID-19, se encontró que suelen observarse reacciones psicológicas agudas que pueden prevalecer a largo plazo, derivado del estado emocional ocasionado por el confinamiento que favorece que las personas sean más propensas a presentar trastornos de ansiedad, de sueño, depresión e incluso estrés agudo (Asmundson \& Taylor, 2020). Así mismo, un estudio realizado en China con estudiantes universitarios se evalúo la ansiedad, miedo y preocupación por el confinamiento, se encontró que los principales factores que incrementan los trastornos de ansiedad se refieren a la cercanía de las personas con pacientes positivos de coronavirus y los efectos económicos de la pandemia (Cao et al., 2020).

Otra de las emociones que se manifiesta es el temor al contagio y a morir por contagio, derivándose en confusión, frustración e ira, la cual se ve magnificada por la sobreinformación y las falsas noticias, así como el aburrimiento ocasionado por el aislamiento, dichas variables fueron encontradas por Brooks et al. (2020) en su estudio metaanalítico sobre los impactos psicológicos que ocurren en los periodos de cuarentena. Además, se consideraron entre las variables que generan temor, las dificultades o pérdidas financieras que genera la situación y la preocupación por la discriminación, en caso de ser positivo para la infección o pertenecer al equipo de salud (Duan \& Zhu, 2020; Lima et al., 2020; Rubin \& Wessley, 2020). Significativamente se han encontrado 
comorbilidades psiquiátricas como la depresión, ansiedad, ataques de pánico, síntomas psicosomáticos y síntomas de estrés postraumático que puede conducir a delirios, psicosis y eventualmente al suicidio (Ho et al., 2020), así como una menor percepción de una calidad de vida favorable (Li et al., 2020).

Otro de los efectos de la cuarentena por la pandemia del COVID-19, hace referencia al cierre de escuelas y la impartición de clases en línea, lo que incrementa el estrés de los padres, así como los problemas emocionales de niños y adolescentes. Los factores estresantes antes mencionados como la duración prolongada del confinamiento, el miedo a la infección, la frustración y el aburrimiento, la información inadecuada, y aunado en niños y adolescentes la falta de contacto con los compañeros de clase, amigos y maestros, pueden tener efectos aún más problemáticos en esta población que en los adultos (Wang et al., 2020). Durante el confinamiento en el hogar, los padres son el recurso más importante para que los niños expresen sus emociones y se comuniquen asertivamente. La comunicación es la clave para identificar problemas físicos y psicológicos y proveer de herramientas para que los niños se comporten saludablemente durante la pandemia.

Debido a las consecuencias de la pandemia del coronavirus y las implicaciones del confinamiento en la salud mental de la población, es importante que los sistemas de salud centren sus esfuerzos en la evaluación oportuna de la población para determinar los efectos en la salud mental derivados de la pandemia y de la cuarentena. Además, se debe poner especial atención en la población vulnerable, por ejemplo, las personas con psicopatología previa, los niños y niñas, cuidadores y personas contagiadas por el coronavirus, familiares y personas cercanas a enfermos de coronavirus, así como el personal de salud en primera línea de atención. Es importante considerar que cualquier intervención psicológica debe planificarse y coordinarse junto con todos los actores implicados principalmente con los profesionales de atención primaria y de las unidades especializadas de salud mental (Inchausti et al., 2020). También debe evitarse que se presente una sobreoferta de asistencia psicológica, la cual debe hacerse de manera coordinada y con los profesionales capacitados para evitar efectos iatrogénicos (Loewenstein, 2018; Ogden, 2019).

Los esfuerzos para la atención psicológica oportuna deben encaminarse hacia la formación de equipos de salud mental de intervención en crisis, comunicación online, intervenciones psicológicas vía remota y el uso de instrumentos breves de detección de problemas de salud mental a través de plataformas digitales, con el propósito de reducir el riesgo de contagios por el virus en la atención cara a cara. Las plataformas online pueden facilitar la identificación de problemas de salud mental y su atención oportuna para evitar el escalamiento a problemas severos. Los programas deben dirigirse a la modificación de actitudes, creencias y comportamientos que permitan la adopción de medidas de autocuidado.

En estos momentos las tecnologías de comunicación se convierten en el principal recurso para la evaluación y el asesoramiento psicológico, para 
los profesionales de salud, pacientes de COVID-19, familiares y sociedad en general, como estrategia para mitigar el impacto emocional y facilitar el retorno a la vida habitual en condiciones seguras.

Las tecnologías de información y comunicación han adquirido mayor aplicabilidad en el cuidado de la salud gracias a que cada año son más las personas que tienen acceso a un dispositivo con conexión a internet, el $76 \%$ de la población urbana en México se considera usuaria de internet, de los cuales el 95\% accede desde un smartphone (INEGI, 2020).

En el terreno de la salud mental el uso de las tecnologías de la información y la comunicación no es un tema nuevo ya que desde el año de 1961 (Wittson et al., 1961) se utilizó la videoconferencia como recurso para el tratamiento de pacientes psiquiátricos. Así mismo en el año 1966, Weizenbaum se logró la programación de un chatbot que era capaz de reconocer palabras claves en la conversación y simulaba las respuestas que haría un terapeuta. En una revisión llevada a cabo por Soto-Pérez et al. (2013) se identifican las publicaciones realizadas con el tema de ciberterapias, encontrando 621 dedicados al biofeedback, 616 a tratamientos cognitivos, 489 a adolescentes, 465 dedicados a la rehabilitación y 412 a tratamientos de tipo conductual.

Por lo tanto, el objetivo de la presente investigación se centró en reportar los principales motivos de consulta mediante la evaluación de problemas de salud mental vía plataformas digitales, así como identificar el efecto de la pandemia y el confinamiento en la salud mental de la población de Aguascalientes.

\section{Método}

La presente investigación se trata de un estudio cuantitativo de tipo descriptivo transversal con recogida de datos previo y durante la cuarentena.

\section{Participantes}

En esta investigación se trabajó con la información proporcionada por las personas usuarias de la Línea VIVE referidos por el chatbot que se encuentra en la página de la Secretaría de Salud del Estado de Aguascalientes y en sus diferentes redes sociales. La Línea Vive es un servicio de atención psicológica vía remota 24/7, y para esta investigación se tomaron los datos de 4,344 personas que llamaron a este servicio durante el periodo del 7 de enero y el 30 de mayo de 2020. El 54\% de las personas atendidas en la Línea Vive fueron mujeres y el $46 \%$ hombres. Los principales rangos de edad de los usuarios fueron de los 12 a 18 años y de los 25 a 35 años con el 70.1\% del total de la muestra (véase Figura 1). 
Figura 1

Rangos de edad de las personas atendidas por el chatbot de la Linea VIVE

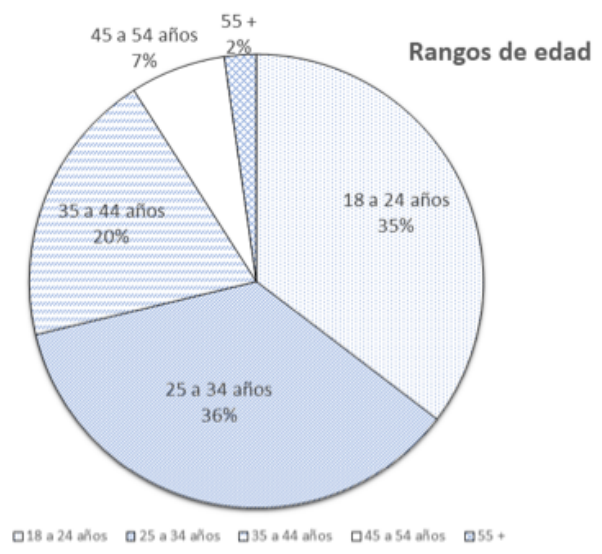

Figure 1

\section{Instrumentos}

Inventario de ansiedad de BECK (Beck, Epstein et al., 1988)

Consta de 21 ítems, con 4 opciones de respuesta que van de poco o nada a severamente. El Inventario fue estandarizado por Jurado et al. (1998) en población mexicana, el cual presentó una confiabilidad alfa de cronbach $\alpha=.87$. Mide ansiedad subjetiva, ansiedad neurofisiológica, ansiedad autónoma y pánico.

Inventario de Depresión de BECK II (Beck, Ward et al., 1961)

El BDI consta de 21 ítems que evalúan fundamentalmente los síntomas clínicos de melancolía y los pensamientos intrusivos presentes en la depresión. Este instrumento fue adaptado a la población mexicana por González et al. (2015) obteniendo una confiabilidad de alfa de cronabach $\alpha=.93$

Escala Revisada de Impacto del Estresor (Weiss, 2007)

Esta escala está compuesta por 22 items y tiene como objetivo evaluar el malestar subjetivo que acompaña y sigue a las experiencias estresantes y/o traumáticas. En una investigación empleando el instrumento en población mexicana se obtuvo una confiabilidad alfa de cronbach $\alpha$ $=.88$ (Hernández-Moreno \& Landero-Hernández, 2015). Para esta investigación se realizó una adaptación de los items relacionándolos con la pandemia del coronavirus.

Escala de Riesgo Suicida de Plutchik (Plutchik \& Van Praag, 1989)

Instrumento de 15 items diseñado para evaluar riesgo suicida, que incluye cuestiones relacionadas con intentos autolíticos previos, intensidad de la ideación actual, sentimientos de depresión y desesperanza, y otros aspectos relacionados con las tentativas de suicidio. En la adaptación realizada para población mexicana se obtuvo una 
confiabilidad alfa de cronbach $\alpha=.88$ (Santana-Campas \& Santoyo, 2018).

\section{Cuestionario de Violencia}

Es un cuestionario diseñado ad hoc para esta investigación que consiste en una herramienta para la detección de violencia psicológica, física y sexual a través de 10 items. Este cuestionario forma parte del expediente electrónico que utiliza la Secretaría de Salud del Estado de Aguascalientes.

Cuestionario de Abuso de Drogas - CAD 20 (Skinner, 1982)

Instrumento de 20 items que proporciona un índice de rango de problemas asociadas con el abuso de drogas. El instrumento se adaptó a la población mexicana por De las Fuentes y Villalpando (2001) obteniendo un alfa de cronbach de 0.95 .

Prueba de identificación de los Trastornos por Uso de Alcohol-AUDIT (Babor et al., 1992)

Cuestionario de 10 items que permite identificar a las personas con un consumo de alcohol perjudicial o de riesgo. Las aplicaciones realizadas en México han reportado una confiabilidad alfa de cronbach superior al 0.85 .

\section{Procedimiento de muestreo}

El 7 de enero de 2020 la Secretaría de Salud del Estado de Aguascalientes implementó la herramienta del chatbot en facebook, que consiste en una plataforma para evaluar voluntariamente a los visitantes que interactúen en el chat una serie de variables psicológicas y ofrecer una inmediata retroalimentación de los niveles de ansiedad, depresión, violencia, consumo de sustancias y riesgo suicida. Los participantes que interactúan con el chatbot cuentan con la disponibilidad de realizar una llamada para atención psicológica o bien elegir el instrumento de evaluación que deseen contestar, al tratarse de mensajes privados se cuenta con privacidad de la información, además las respuestas de los participantes son registradas en una base de datos de acceso limitado a autoridades de la Secretaría de Salud del Estado de Aguascalientes.

Los participantes no obtuvieron un pago, pero sí la retroalimentación sobre su nivel de riesgo, si la persona es evaluada como de riesgo alto o muy alto de presentar problemas de salud mental. El chatbot además, le da la opción de comunicarse en ese momento a la Línea Vive, o en su defecto, el sistema le recuerda constantemente que entable comunicación para recibir el apoyo de un profesional de la salud mental.

La métrica del chatbot permite tener las impresiones, es decir el número de veces que el anuncio aparece en las plataformas digitales; el alcance, que significa el número de personas que ven de manera efectiva el anuncio; y los mensajes, determinado por el número de veces que el chatbot interactúa con los usuarios de la plataforma. En acuerdo con las diferentes fases en que se han establecido para la pandemia del coronavirus en México, se realizaron diferentes cortes de los instrumentos cargados en el chatbot, considerando su métrica, el número de personas que hacen contacto con la línea vive, y el número de personas que son referidas a recibir tratamiento psicológico. 
Cabe mencionar que, desde el 7 de enero de 2020, el chatbot interactúa con los usuarios con seis de los instrumentos de evaluación propuestos en esta investigación, los cuales miden ansiedad, depresión, riesgo suicida, consumo de sustancias y violencia. A partir del 25 de abril se cargó el instrumento de evaluación del impacto estresor. Para obtener los resultados se realizó un análisis a partir de las diferentes fases de la pandemia del coronavirus, es decir del 7 de enero al 23 de marzo se registró el número de personas que contestaron los diferentes instrumentos de evaluación, el cual fue considerado como línea base. A partir del 24 de marzo y hasta el 21 de abril, periodo de la fase dos de la pandemia, se analizó el impacto de la pandemia del coronavirus en estas variables psicológicas, así como también durante la fase tres de la pandemia que inició el 22 de abril y finalizó el 30 de mayo. En esta última fase se agregó el análisis de la Escala del Impacto Estresor por COVID-19, las fechas y cohortes se muestran en la siguiente tabla.

Tabla 1

Tabla 1

Fechas de recopilación de los datos

\begin{tabular}{ccc} 
Cohorte & Fecha de recopilación & Ubicación \\
\hline Línea Base & $7 / 01 / 2020$ al 23/03/2020 & Chatbot Facebook \\
Fase 2 & $24 / 03 / 2020$ al 21/04/2020 & Chatbot Facebook \\
Fase 3 & $22 / 04 / 2020$ al 30/05/2020 & Chatbot Facebook
\end{tabular}

\section{Consideraciones éticas}

El presente proyecto fue autorizado por la dirección de investigación de la Universidad Autónoma de Aguascalientes, en donde se determinó la viabilidad ética de las evaluaciones psicológicas vía remota. Se acordó evaluar a los participantes y retroalimentar sobre su nivel de riesgo si ellos autorizaban responder el cuestionario, quienes desde el comienzo de la interacción firman un consentimiento informado. Aquellos que realizaban la llamada a la Línea Vive, se les brindó atención psicológica gratuita. No existieron pagos monetarios por la participación.

\section{Análisis de datos}

Los datos se analizaron obteniendo las puntuaciones de cada una de las pruebas adaptadas a la población mexicana y devolviendo el grado de riesgo a los participantes. Posteriormente se realizó un registro de frecuencias de los motivos de llamada en la atención psicológica remota por día, obteniendo la tasa de atención diaria. Finalmente, se aplicó el análisis de datos no paramétricos para datos independientes Kruskall 
Wallis para determinar las diferencias entre las pruebas psicológicas aplicadas.

\section{Resultados}

Como primer punto es importante mencionar que, del 7 de enero al 30 de mayo de 2020, se dieron 5,593,233 de impresiones en el chatbot, con un alcance de 4,383,142 personas. El chatbot tuvo interacción a través de mensajes con 48,072 personas, esto significa que el $1.1 \%$ de las personas que ven las publicaciones en las diferentes plataformas digitales inicia con el proceso de llenado de alguno de los diferentes instrumentos de evaluación. Otro resultado relevante es que el $20 \%$ de las personas que interactúan a través de mensajes con el chatbot, concluyen con el proceso de evaluación, en este caso 9,615 personas, de las cuales 4,344 se contactaron a la Línea Vive por obtener un resultado que refiere un problema de salud mental o consumo problemático de sustancias, es decir el $20 \%$ de las personas que concluyen con el llenado de alguno de los instrumentos. En la siguiente figura se puede observar a los participantes que continúan la interacción con el chatbot hasta ser atendidos telefónicamente.

Figura 2

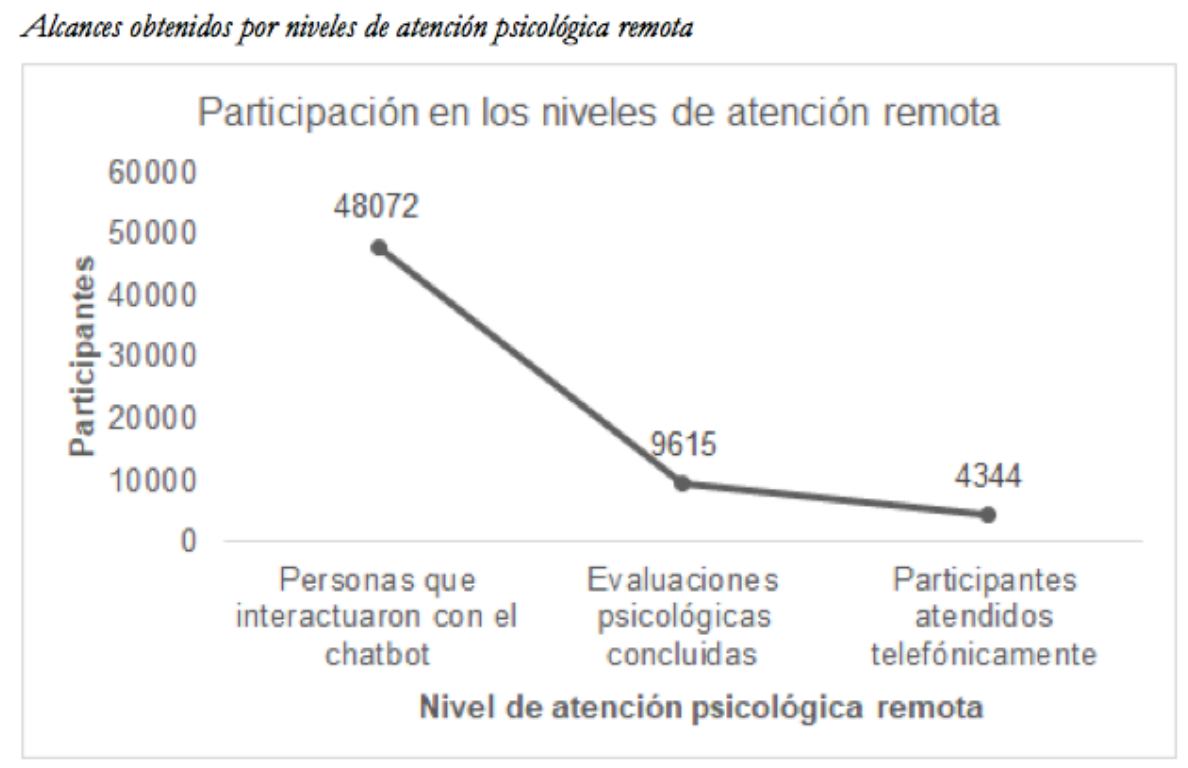

Figura 2

En el análisis que se muestra a continuación se presenta el porcentaje de personas que obtuvo un puntaje de riesgo alto o muy alto en cada uno los diferentes instrumentos de evaluación, así como el total de llamadas a la Línea Vive en cada una de las fases, sin embargo, para mostrar diferencias entre las fases, en este último rubro se obtuvo la tasa de llamadas diarias para cada una de las problemáticas registradas. 
Durante la fase de línea base fueron canalizados por el chatbot a la Línea Vive 1,988 personas, por presentar algún problema de salud mental o de consumo de sustancias. El 31.36\% de las personas presentó comportamiento de riesgo suicida, el $28.86 \%$ presentó problemas por el consumo de drogas o alcohol, el 20.46\% refirió síntomas de depresión, el $19.1 \%$ presentó ansiedad, y finalmente el $0.22 \%$ problemas de violencia intrafamiliar.

En la fase dos de la pandemia fueron atendidas en la Línea Vive 943 personas referidas por el chatbot, debido a que fueron evaluados con problemas del comportamiento. El porcentaje de personas que presentó comportamiento de riesgo suicida fue de $25.87 \%$, el $32.45 \%$ presentó problemas por el consumo de drogas o alcohol, el $22.07 \%$ refirió síntomas de depresión, el $18.14 \%$ presentó ansiedad, y finalmente el $1.47 \%$ problemas de violencia intrafamiliar.

Durante la fase tres de la pandemia se atendieron 1,413 personas en la Línea Vive, el porcentaje de personas por consumo de sustancias fue del $30.51 \%$, comportamiento de riesgo suicida $26.06 \%$, depresión $23.73 \%$, ansiedad $17.72 \%$, y violencia intrafamiliar $1.98 \%$. También, en esta fase es importante mencionar que el $10 \%$ de las personas atendidas mostró índices elevados de estrés en relación con el confinamiento derivado de la pandemia.

Dado que las diferentes fases en las cuales se está realizando las mediciones no son homogéneas en el número de días, se hizo una proporción del número de personas atendidas por día, obteniendo así la tasa diaria para cada una de las problemáticas de salud mental evaluadas en cada una de las fases (véase Figura 2). Así, durante la fase de línea base la tasa de atenciones diarias fue de 26.16, y como se muestra en la Figura 3, por problemas de ansiedad la tasa diaria fue de 5.02 personas, por depresión 5.36 personas, por violencia 0.06 personas, por comportamiento suicida 8.21 personas y por problemas por el consumo de sustancias fue de 7.51 personas. En la fase dos de la pandemia la tasa de atenciones diarias para problemas de ansiedad fue de 5.9 personas, por depresión 7.18 personas, por violencia 0.48 personas, debido a riesgo por comportamiento suicida fue de 8.42 personas y por problemas por el consumo de sustancias fue de 10.56 personas; en esta fase la tasa de atenciones diarias fue de 32.54 personas. Finalmente, en la fase 3 las atenciones diarias fueron en promedio 36.25 personas, de las cuales por problemas de ansiedad la tasa diaria fue de 6.42 personas, por depresión 8.6 personas, por violencia 0.72 personas, por comportamiento suicida 9.45 personas y por problemas por el consumo de sustancias fue de 11.06 personas. Análisis no paramétricos para datos independientes Kruskall Wallis muestran diferencias estadísticas significativas entre las fases para los problemas de ansiedad, depresión, consumo de sustancias, comportamiento suicida, pero no mostró diferencias para la violencia intrafamiliar entre las fases, tal como se muestra en la siguiente tabla. 
Tabla 2

Tabla 2

Resultados de la prueba Kruskall Wallis

\begin{tabular}{|c|c|c|c|c|}
\hline Ansiedad & Depresión & $\begin{array}{l}\text { Consumo de } \\
\text { sustancias }\end{array}$ & $\begin{array}{l}\text { Comportamiento } \\
\text { suicida }\end{array}$ & $\begin{array}{c}\text { Violencia } \\
\text { intrafamiliar }\end{array}$ \\
\hline $\begin{array}{c}\mathrm{X} 2(2)=8.06 \\
\mathrm{p}<0.05\end{array}$ & $\begin{array}{c}X 2(2)=12.75 \\
p<0.01\end{array}$ & $\begin{array}{c}\mathrm{X} 2(2)=13.57 \\
\mathrm{p}<0.01\end{array}$ & $\mathrm{X} 2(2)=7.78, \mathrm{p}<0.05$ & $\begin{array}{c}X 2(2)=4.35 \\
p>0.05\end{array}$ \\
\hline
\end{tabular}

Del mismo modo se muestra gráficamente la tasa de llamadas diarias atendidas por categorización de motivos de llamada, antes de la pandemia y durante las fases 2 y 3 de la pandemia anunciadas por el Gobierno de México (2020).

Figura 3

Categorización de las llamadas recibidas a la linea vive

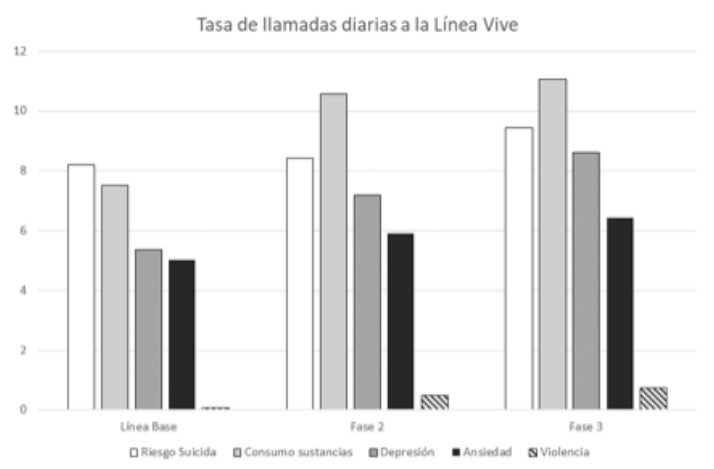

Nota. Comparación de la frecuencia de problemas de salud mental atendidos antes y durante la cuarentena.

Figura 3

Finalmente, es importante mencionar que el $40 \%$ de las personas que contactaron a la Línea Vive fueron canalizadas para recibir un proceso psicoterapéutico.

\section{Discusión}

Es importante mencionar que los datos presentan un sesgo de alcance poblacional dado que existe población que no fue evaluada por limitaciones en acceso a internet o disponibilidad de dispositivos tecnológicos por lo que la interpretación de los datos debe manejarse con cautela al momento de generalizarlos a una población total, dichas consideraciones se están tomando en cuenta para aplicaciones posteriores 
en los que se incluyan participantes de la mayoría de los estratos y condiciones socioeconómicas.

La enfermedad por coronavirus COVID-19 ha dejado de manifiesto que preservar la salud física es determinante para las personas y para los sistemas de salud de los estados, sin embargo, los efectos a la salud mental en muchas comunidades han sido devastadores, tal como lo reportan Torales et al. (2020) las consecuencias son observables en el incremento de síntomas encontrados en el presente estudio. Las personas suelen reaccionar con miedo e incertidumbre ante las diferentes fases de la pandemia, derivándose en percepciones distorsionadas, principalmente baja percepción del riesgo, dando como resultado consumo de sustancias adictivas, violencia, síntomas de ansiedad, estrés y depresión (Shigemura et al., 2020); incrementando el riesgo de suicidio (Jobes et al., 2020).

En esta investigación se pudo identificar un incremento en el número de personas que solicitan los servicios psicológicos vía remota (Línea Vive), principalmente para la atención de la ansiedad, depresión, consumo de sustancias y riesgo suicida, los cuales se elevaron en cada una de las fases estudiadas.

En el caso de la ansiedad se observó el mayor incremento de la fase dos a la fase tres de la pandemia, que se relaciona con el incremento de los contagios y muertes en México y en el Estado de Aguascalientes, y respecto a la depresión el principal incremento se dio al inicio de la fase dos de la pandemia. Lo anterior coincide con lo encontrado por Asmundson y Taylor (2020), en su estudio sobre ansiedad ante los confinamientos a nivel mundial, la ansiedad se ve relacionada con tres condiciones; la sobreinformación, el miedo al contagio y el confinamiento. Este trastorno hace referencia a cambios o sensaciones corporales que son interpretados como síntomas de alguna enfermedad, en este caso del COVID-19, por lo que las personas creen que han contraído la enfermedad (Asmundson \& Taylor, 2020). También, es importante mencionar que cuando la gente experimenta los efectos del encierro se elevan los niveles de ansiedad y depresión, incluso se espera se incrementen aún más cuando se prolonga el aislamiento social, los resultados de nuestro estudio coinciden con los encontrados por Ozamiz-Etxebarria et al. (2020) en el que se midieron los niveles de estrés, ansiedad y depresión durante el confinamiento por COVID-19 en España. Aunado a esto, los mitos y la desinformación en las redes sociales provocan incertidumbre y ambigüedad en los comportamientos que se debe adoptar para hacer frente a la pandemia y el confinamiento, lo que hace más errático adoptar comportamientos saludables (Bao et al., 2020).

Estas mismas condiciones pueden explicar la relación con diversos problemas de salud mental, como ya se mencionó la ansiedad y la depresión, así como el consumo de sustancias adictivas (Alexander, 2012; Bloem et al., 2019; Gage \& Sumnall, 2018). Así, los niveles de ansiedad pueden llevar a ciertas personas a presentar un consumo de drogas, o este puede surgir como una forma de mitigar los efectos del confinamiento y la incertidumbre (Doyle et al., 2019; Rojas-Jara, 2018, 2020). En esta investigación se pudo observar que el consumo de sustancias fue 
el principal problema del comportamiento atendido en la Línea Vive, sin embargo, es de recalcar que antes de la pandemia fue la segunda problemática por lo que la gente contactaba a la Línea Vive, solo por detrás del comportamiento de riesgo suicida. Respecto al riesgo suicida se observó un ligero incremento de la fase dos a la fase tres de la pandemia, el comportamiento suicida, dado los incrementos que se han observado en el Estado de Aguascalientes en los últimos años, en los momentos de la pandemia requiere una especial atención, lo que lleva a diseñar estrategias para continuar con la atención de estas personas y evitar que los problemas de salud mental escalen a comportamiento suicida. Por lo tanto, se deben reforzar los sistemas de evaluación y tratamiento a distancia, que garanticen una atención oportuna a los problemas de salud mental y en especial al riesgo de cometer suicidio.

Finalmente, es importante mencionar la violencia que se genera en los hogares a partir del confinamiento, y aunque en esta investigación no se observó un incremento significativo de las personas que utilizaron los servicios psicológicos vía remota, esto no significa que la violencia, y principalmente hacia la mujer no se presentó, por el contrario, se ha reportado un incremento en otros países como España (Ruiz-Pérez \& Pastor-Moreno, 2020). Lo que deja claro este resultado es la falta de solicitud de apoyo, principalmente, de las mujeres, en tanto la forma más común de violencia es la infligida por la pareja, situación que se agrava durante la pandemia, ya que se debe convivir con el agresor durante el aislamiento, lo que no da oportunidad para denunciar o solicitar apoyo, por lo tanto, se deben desarrollar estrategias de comunicación oportuna para salvaguardar la vida más aún cuando se convive las 24 horas con el agresor (Lorente, 2020).

La cuarentena impactó de manera directa en la salud mental de las personas, muchas de ellas contaron con los recursos psicológicos para enfrentar, tanto la pandemia y el confinamiento de manera satisfactoria sin requerir de un apoyo profesional, otras personas requirieron el apoyo en momentos de crisis, y otro porcentaje menor requirió de un tratamiento psicológico para solucionar afecciones mentales durante esta pandemia. La incertidumbre respecto de la enfermedad y las restricciones temporales a de la libertad de circulación, se suman a la distancia respecto de las familias y amistades, lo cual se relaciona directamente en el estado mental de la población.

Las consecuencias de la pandemia no se deben limitar en el tiempo, ni a los servicios de atención para preservar la salud física, se deben considerar los aportes y recomendaciones de la psicología. Se deben desarrollar programas para detectar y tratar problemas de salud mental, empleando de manera estratégica programas de intervención remota. Es imperante fortalecer los servicios de internet y el uso de teléfonos inteligentes para proveer los servicios de salud mental en línea y proporcionar este tipo de intervención las 24 horas día, todos los días de la semana.

Dentro de las limitaciones que se encuentran en el presente estudio se encuentra como primer punto el alcance a la población económicamente vulnerable, que carece de acceso a servicios de internet y/o dispositivos 
tecnológicos, si bien se tiene un nivel alto de participación, hay sectores de la población a los que no ha llegado la información sobre la atención. Otro punto a considerar es que a pesar del alcance que tiene la plataforma es poco el porcentaje de personas que concluyen las evaluaciones y continúan el proceso hasta la atención psicológica. Por último, se considera que la replicabilidad del estudio será costosa sin una página cuyo alcance sea a nivel estatal y sin un equipo que brinde la atención psicológica a los participantes identificados en alto y muy alto riesgo.

\section{Agradecimientos}

La presente investigación cuenta con el financiamiento por parte de la Universidad Autónoma de Aguascalientes, con el proyecto llamado "Impacto de la atención telefónica sobre los problemas de salud mental y adicciones durante la contingencia de COVID-19", con la clave PIPS20-8C. Los autores declaran no tener conflicto de intereses. Se agradece el apoyo otorgado por parte de la Universidad Autónoma de Aguascalientes y la Secretaria de Salud del Estado de Aguascalientes por los apoyos y facilidades brindados.

\section{Referencias}

Alexander, B. K. (2012). Addiction: The urgent need for a paradigm shift. Substance Use \& Misuse, 47, 1475-1482. https://doi.org/10.3109/10826 084.2012 .705681

Asmundson, G. J., \& Taylor, S. (2020). How health anxiety influences responses to viral outbreaks like COVID-19: What all decision-makers, health authorities, and health care professionals need to know. Journal of Anxiety Disorders, 71, 102211. https://doi.org/10.1016/j.janxdis.2020.102211

Babor, T., de la Fuente, J., Saunders, J., \& Grant, M. (1992). AUDIT: The alcohol use disorders identification test: Guidelines for use in primary health care. Organización Mundial de la Salud.

Bao, Y., Sun, Y., Meng, S., Shi, J., \& Lu, L. (2020). 2019-nCoV epidemic: Address mental health care to empower society. The Lancet, 395(10224), e37-e38. https://doi.org/10.1016/S0140-6736(20)30309-3

BBC. (2020). Coronavirus, México registra el primer muerto por covid-19. https: //www.bbc.com/mundo/noticias-america-latina-51959283

Beck, A. T., Epstein, N., Brown, G., \& Steer, R. (1988). Beck Anxiety Inventory [Database record]. APA PsycTests. https://doi.org/10.1037/t02025-000

Beck, A. T., Ward, C. H., Mendelson, M., Mock, J., \& Erbaugh, J. (1961). An inventory for measuring depression. Archives of General Psychiatry, 4, 561-571. https://doi.org/10.1001/archpsyc.1961.01710120031004

Bloem, O., Bulten, E., \& Verkes, R. (2019). Changes in subjective wellbeing of prisoners on remand.International Journal of Prisoner Health, 15(2), 181-191. https://doi.org/10.1108/IJPH-01-2018-0003

Brooks, S. K., Webster, R. K., Smith, L. E., Woodland, L., Wessely, S., Greenberg, N., \& Rubin, G. J. (2020). The psychological impact of quarantine and how to reduce it: Rapid review of the evidence. Lancet, 
395(10227), 912-920. https://doi.org/10.1016/S0140-6736(20)304608

Caballero-Domínguez, C. C., \& Campo-Arias, A. (2020). Problemas de salud mental en la sociedad: un acercamiento desde el impacto del COVID 19 y de la cuarentena. Duazary, 17(3), 1-3. https://doi.org/10.21676/2389 783X.3467

Cao, W., Fang, Z., Hou, G., Han, M., Xu, X., Dong, J., \& Zheng, J. (2020). The psychological impact of the COVID-19 epidemic on college students in China. Psychiatry Research, 287, 112934. https://doi.org/10.1016/j.psyc hres.2020.112934

De las Fuentes, V. M., \& Villalpando, J. (2001). Adaptación de un instrumento de tamizaje para la población mexicana que consume drogas [Tesis de Licenciatura]. Universidad Nacional Autónoma de México, México.

Doyle, M., Shakeshaft, A., Guthrie, J., Snijder, M., \& Butler, T. (2019). A systematic review of evaluations of prison-based alcohol and other drugs use behavioral treatment for men. Australian and New Zealand Journal of Public Health, 43(2), 120-130. https://doi.org/10.1111/1753-6405.128 84

Duan, L., \& Zhu, G. (2020). Psychological interventions for people affected by the COVID-19 epidemic. The Lancet Psychiatry, 7(4), 300-302. https:// doi.org/10.1016/S2215-0366(20)30073-0

Gage, S., \& Sumnall, H. R. (2018). Rat park: How rat paradise changes the narrative of addiction. Addiction, 114(5), 917-922. https://doi.org/10.11 $11 /$ add. 14481

Gobierno de México. (2020). Jornada Nacional de Sana Distancia. https://www.gob.mx/salud/hospitalgea/documentos/jornada -nacional-de-sana-distancia

González, D. A., Rodríguez, A. R., \& Reyes-Lagunes, I. (2015). Adaptation of the BDI-II in Mexico. Salud Mental, 38(4), 237-244. https://doi.org/10 $.17711 /$ SM.0185-3325.2015.033

Hernández Moreno, F. P., \& Landero Hernández, R. (2015). Aspectos psicosociales relacionados con el TEPT en pacientes con cáncer de mama. Acta de Investigación Psicológica, 5(1), 1881-1891.

Ho, C. S., Chee, C. Y., \& Ho, R. C. (2020). Mental health strategies to combat the psychological impact of COVID-19 beyond paranoia and panic. Annals of the Academy of Medicine, Singapore, 49(3), 155-160.

Inchausti, F., Garcia-Poveda, N. V., Prado-Abril, J., \& Sanchez-Reales, S. (2020). La psicología clínica ante la pandemia COVID-19 en España. Clínica y Salud. Avance Online, 31(2), 105-107. https://doi.org/10.5093/clysa202 0a11

INEGI. (2020). Encuesta Nacional sobre Disponibilidad de Tecnologías de Información en los hogares (ENDUTIH, 2019) en Comunicado de Prensa (103/20). https://www.inegi.org.mx/contenidos/saladeprensa/boletines /2020/OtrTemEcon/ENDUTIH_2019.pdf

Instituto Mexicano del Seguro Social (IMSS). (2020). Mapa en tiempo real de casos de coronavirus por Johns Hopkins School. http://cvoed.imss.gob.mx/ mapa_coronavirus/

Jobes, D. A., Crumlish, J. A., \& Evans, A. D. (2020). The COVID-19 pandemic and treating suicidal risk: The telepsychotherapy use of CAMS. Journal of 
Psychotherapy Integration, 30(2), 226-237. https://doi.org/10.1037/int0 000208

Johns Hopkins University and Medicine. (2020). COVID-19 Dashboard by the Center for Systems Science and Engineering (CSSE). https://coronavirus.j hu.edu/map.html

Jurado, S., Villegas, M. E., Méndez, L., Rodríguez, F., Loperena, V., \& Varela, R. (1998). La estandarización del Inventario de Depresión de Beck para los residentes de la Ciudad de México. Salud Mental, 21(3), 26-31.

Li, S., Wang, Y., Xue, J., Zhao, N., \& Zhu, T. (2020). The impact of COVID-19 epidemic declaration on psychological consequences: A study on active Weibo users. International Journal of Environmental Research and Public Health, 17(6), 2032. https://doi.org/10.3390/ijerph17062032

Lima, C. K. T., de Medeiros Carvalho, P. M., Lima, I. D. A. S., de Oliveira Nunes, J. V. A., Saraiva, J. S., de Souza, R. I., ... \& Neto, M. L. R. (2020). The emotional impact of Coronavirus 2019-nCoV (new Coronavirus disease). Psychiatry Research, 287, 112915. http://dx.doi.org/10.1016/j.psychres.2 020.112915

Loewenstein, R. J. (2018). Dissociation debates: Everything you know is wrong. Dialogues in Clinical Neuroscience, 20(3), 229-242. https://doi.org/10.31 887/DCNS.2018.20.3/rloewenstein

Lorente, M. (2020). Violencia de género en tiempos de pandemia y confinamiento. Revista Española de Medicina Legal, 46(3), 139-145. http s://doi.org/10.1016/j.reml.2020.05.005

Ogden, J. (2019). Do no harm: Balancing the costs and benefits of patient outcomes in health psychology research and practice. Journal of Health Psychology, 24(1), 25-37. https://doi.org/10.1177/1359105316648760

Organización Mundial de la Salud. (2020). Coronavirus disease (COVID-19). h ttps://www.who.int/emergencies/diseases/novel-coronavirus-2019/

Ozamiz-Etxebarria, N., Dosil, M., Picaza, M., \& Idoiaga, N. (2020). Níveis de estresse, ansiedade e depressão na primeira fase do surto de COVID-19 em uma amostra no norte da Espanha. Cad. Saúde Pública, 36(4). http://dx. doi.org/10.1590/0102-311X00054020

Plutchik, R., \& Van Praag, H. (1989). The measurement of suicidality, aggressivity and impulsivity. Progress in Neuro-Psychopharmacology \& Biological Psychiatry, 13(Suppl), S23-S34. https://doi.org/10.1016/027 8-5846(89)90107-3

Rojas-Jara, C. (2018). Innovation in the field of drugs: the need to rethink the use, the user, and the psychological treatment. En J. C. Penagos-Corso \& M. A. Padilla (Eds.), Challenges in creativity \& psychology for the XXI century (pp. 259-271). UDLAP/Universidad de Guadalajara.

Rojas-Jara, C. (2020). Cuarentena, aislamiento forzado y uso de drogas. Cuadernos de Neuropsicologia/Panamerican Journal of Neuropsychology, 14(1), 24-28.

Rubin, G. J., \& Wessely, S. (2020). The psychological effects of quarantining a city. British Medical Journal, 368, 313. http://dx.doi.org/org/10.1136/b mj.m313

Ruiz-Pérez, I., \& Pastor-Moreno, G. (2020). Medidas de contención de la violencia de género durante la pandemia de COVID-19. Gaceta Sanitaria. https://doi.org/10.1016/j.gaceta.2020.04.005 
Santana-Campas, M. A., \& Santoyo, F. (2018). Propiedades psicométricas de la escala riesgo suicida de Plutchik en una muestra de jóvenes mexicanos privados de la libertad. Avances en Psicología, 26(1), 57-64. https://doi.or g/10.33539/avpsicol.2018.v26n2.1127

Shigemura, J., Ursano, R. J., Morganstein, J. C., Kurosawa, M., \& Benedek, D. M. (2020). Public responses to the novel 2019 coronavirus (2019-nCoV) in Japan: Mental health consequences and target populations. Psychiatry and Clinical Neurosciences, 74(4), 281-282. https://doi.org/10.1111/pcn .12988

Skinner, H. A. (1982). The drug abuse screening test. Addictive Behaviors, 7(4), 363-371. https://doi.org/10.1016/0306-4603(82)90005-3

Soto-Pérez, Felipe, Bernate Navarro, M., González-Palau, Toribio-Guzmán, J. M., Aguado, Y., \& Franco-Martín, M. (2013). A brief history of cyber therapies: Mental health \& technologies. Revista Argentina de Ciencias del Comportamiento, 5, 361-363.

Torales, J., O’Higgins, M., Castaldelli-Maia, J. M., \& Ventriglio, A. (2020). The outbreak of COVID-19 coronavirus and its impact on global mental health. International Journal of Social Psychiatry, 66(4), 317-320. https:/ /doi.org/10.1177/0020764020915212

Wang, G., Zhang, Y., Zhao, J., Zhang, J., \& Jiang, F. (2020). Mitigate the effects of home confinement on children during the COVID-19 outbreak. The Lancet, 395(10228), 945-947. https://doi.org/10.1016/S0140-6736(20) 30547-X

Weiss, D. S. (2007). The impact of event scale: Revised. En J. P. Wilson \& C. S. K. Tang (Eds.), International and cultural psychology. Cross-cultural assessment of psychological trauma and PTSD (pp. 219-238). Springer Science + Business Media. https://doi.org/10.1007/978-0-387-70990-1 -10

Wittson, C. L., Affleck, D. C., \& Johnson, V. (1961). Two-way television in group therapy. Mental Hospitals, 12(1), 22-23. https://doi.org/10.1176/ ps.12.11.22

Weizenbaum, J. (1966). Eliza-a computer program for the study of natural language communication between man and machine. Communications of the ACM, 9(1), 36-45. https://doi.org/10.1145/365153.365168 